Corrigendum

\title{
Corrigendum to: "Striatal changes in Parkinson disease: An investigation of morphology, functional connectivity and their relationship to clinical symptoms" [Psychiatry Research: Neuroimaging. Volume 275 (May 2018),
} Pages 5-13]

\author{
Conor Owens-Walton ${ }^{\mathrm{a}, *}$, David Jakabek ${ }^{\mathrm{b}}$, Xiaozhen $\mathrm{Li}^{\mathrm{c}, \mathrm{d}}$, Fiona A. Wilkes ${ }^{\mathrm{a}}$, Mark Walterfang ${ }^{\mathrm{e}, \mathrm{f}}$, \\ Dennis Velakoulis ${ }^{\mathrm{e}}$, Danielle van Westen ${ }^{\mathrm{g}, \mathrm{h}}$, Jeffrey C.L. Looi ${ }^{\mathrm{a}, \mathrm{e}}$, Oskar Hansson ${ }^{\mathrm{d}, \mathrm{i}}$ \\ ${ }^{\text {a }}$ Research Centre for the Neurosciences of Ageing, Academic Unit of Psychiatry and Addiction Medicine, School of Clinical Medicine, Australian National University \\ Medical School, Canberra, Australia \\ ${ }^{\mathrm{b}}$ Graduate School of Medicine, University of Wollongong, Wollongong, Australia \\ ${ }^{\mathrm{c}}$ Division of Clinical Geriatrics, Centre for Alzheimer Disease Research, Department of Neurobiology, Care Sciences and Society (NVS), Karolinska Institute, Huddinge, \\ Sweden \\ ${ }^{\mathrm{d}}$ Department of Clinical Sciences, Lund University, Malmö, Sweden \\ ${ }^{\mathrm{e}}$ Neuropsychiatry Unit, Royal Melbourne Hospital, Melbourne Neuropsychiatry Centre, University of Melbourne \& Northwestern Mental Health, Melbourne, Australia \\ ${ }^{\mathrm{f}}$ Florey Institute of Neurosciences and Mental Health, University of Melbourne, Melbourne, Australia \\ ${ }^{g}$ Center for Medical Imaging and Physiology, Skåne University Hospital, Lund, Sweden \\ ${ }^{\mathrm{h}}$ Diagnostic Radiology, Department of Clinical Sciences, Lund University, Lund, Sweden \\ ${ }^{\mathrm{i}}$ Memory Clinic, Skåne University Hospital, Malmö, Sweden
}

The authors regret a mistake in the Discussion section of this article, where we stated: "As lateralised analysis only demonstrated volumetric reductions of the right caudate nucleus and the left putamen, our results only partially support our hypotheses." The correct statement is "As lateralised analysis only demonstrated volumetric reductions of the left caudate nucleus and the right putamen, our results only partially support our hypotheses."

The authors would like to apologise for any inconvenience caused.

DOI of original article: https://doi.org/10.1016/j.pscychresns.2018.03.004

* Corresponding author.

E-mail address: conor.owens-walton@anu.edu.au (C. Owens-Walton). 